\title{
Development of Sandglass Shape FBG Sensor to Reduce Cross Sensitivity Effect
}

\author{
Bo Zhang \\ Xian University of Science and Technology, No. 58 Yan Ta Street, Xian, Shanxi 710054, China \\ Correspondence should be addressed to Bo Zhang; xiankjdx2012@163.com
}

Received 24 April 2013; Revised 22 May 2013; Accepted 31 May 2013

Academic Editor: Augusto Belendez

Copyright ( 2013 Bo Zhang. This is an open access article distributed under the Creative Commons Attribution License, which permits unrestricted use, distribution, and reproduction in any medium, provided the original work is properly cited.

Fiber Bragg grating (FBG) sensors have received considerable attention in applications of temperature, axial strain, and transverse pressure measurement. However, the cross-sensitivity of strain and temperature measurement is the key problem of FBG sensors. In this paper, a sandglass shape FBG is proposed to divide cross-sensitivity of temperature and transverse pressure. The principle and structure of sandglass FBG are introduced, and the experiment results show cross-sensitivity avoidable in the transverse pressure sensor.

\section{Introduction}

Fiber Bragg grating (FBG) has unique advantages compared to metal sensors such as immunity to electromagnetic interference, compact size, potential low cost, and the possibility of distributed measurement over a long distance, which have been reported as sensors for many measurements including temperature, strain, vacuum, and refractive index. FBG sensors have received great attention for applications in modern stress measurement and optical distribution sensing networks [1]. However, the cross-sensitivity effects of FBGs, like force, displacement, or pressure effects tangled with temperature effect, make it difficult to separately determine the temperature and other parameters by measurement of the wavelength shift of single-FBG sensor, which reduce the utilization value in the field $[2,3]$. In this paper, an approach to resolve the cross-sensitivity of FBGs is proposed and demonstrated, which is using the sandglass coat shape of FBG with and polyimide polymers to resolve the cross-sensitivity problem during the demodulation. As an application of this approach, simultaneous measurement of axial strain, and temperature with sandglass FBG coatings is reported.

\section{Cross-Sensitivity Effect of FBGs}

As shown in Figure 1, an FBG of $1 \mathrm{~cm}$ in the grating length was inscribed on a standard telecommunication single-mode optical fiber (corning SMF-28).
The Bragg resonance wavelength of a FBG, $\lambda_{B}$, which is the center wavelength of light back reflected from the grating, depends on the effective index of refraction of the core $\left(n_{\text {eff }}\right)$ and the periodic spacing of the grating $(\Lambda)$ through the relation $\lambda_{B}=2 n_{\text {eff }} \Lambda$. Parameters such as $n_{\text {eff }}$ and $\Lambda$ are affected by changes in strain and temperature. The shift in the Bragg resonance wavelength $\Delta \lambda_{B}$ due to the changes in the temperature and strain can be described by

$$
\Delta \lambda_{B}=2\left(\Lambda \frac{\partial n}{\partial T}+n \frac{\partial \Lambda}{\partial T}\right) \Delta T+2\left(\Lambda \frac{\partial n}{\partial l}+n \frac{\partial \Lambda}{\partial l}\right) \Delta l
$$

The first term in (1) represents the temperature effect on the FBG and the second term describes the strain effect. The fractional wavelength shift corresponding to a temperature change $\Delta T$ as

$$
\Delta \lambda_{B, T}=\lambda_{B}(\alpha+\xi) \Delta T
$$

where $\alpha$ is the thermal expansion coefficient of the fiber normally is $0.55 \times 10^{-6}{ }^{\circ} \mathrm{C}^{-1}$ for silica cladding. The quantity $\xi$ represents the thermooptic coefficient, which is $8.6 \times$ $10^{-6}{ }^{\circ} \mathrm{C}^{-1}$ for a germanium-doped silica core. The term related to the strain effect can be expressed as

$$
\Delta \lambda_{B, S}=\lambda_{B}\left(1-\widehat{P}_{e}\right) \Delta \varepsilon,
$$




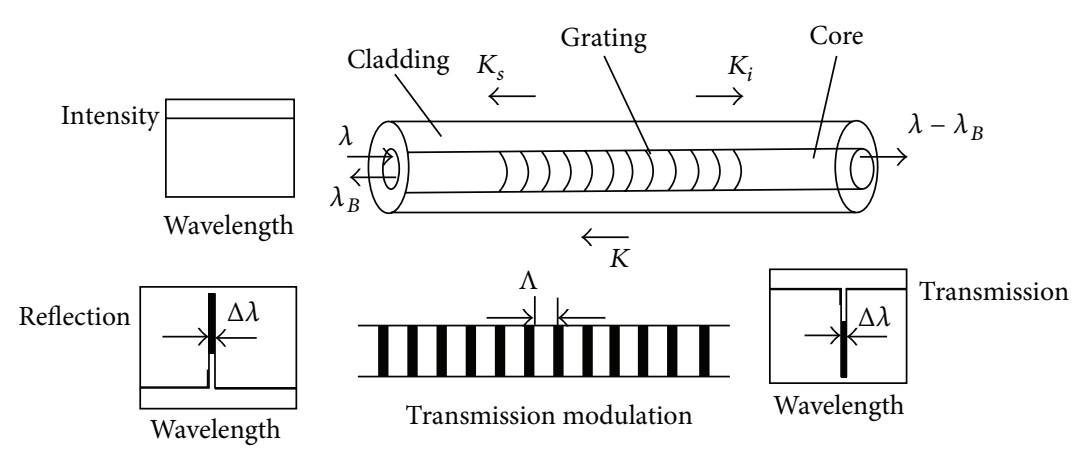

FIGURE 1: Refractive index distributions of FBG and transmission and reflection characters.
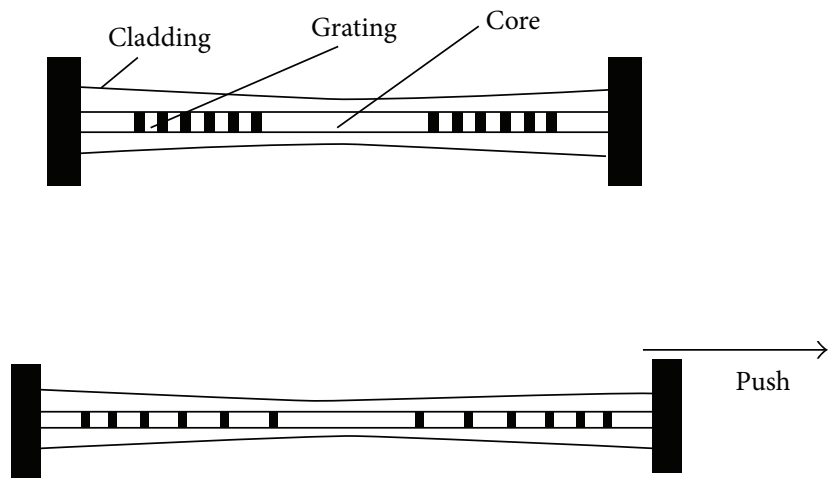

FIGURE 2: Sketch of sandglass FBG and applied stress.

where $\widehat{P}_{e}$ is an effective photoelastic constant with a value of 0.22 for fused silica:

$$
\Delta \lambda_{B}=\Delta \lambda_{B, T}+\Delta \lambda_{B, S}
$$

In (4) shown, the wavelength shifting is made by the temperature and strain changing together, so the division of cross-sensitivity of strain and temperature is one of the key technologies of the FBG sensor in the application $[4,5]$.

\section{Working Principle and Fabrication of Sandglass Shape FBGs}

In this paper, the sandglass shape FGB is proposed to avoid cross-sensitivity effect between strain and temperature. The main principle is to make nonuniform strain on the grating pattern, whose results show that the grating reflection bandwidth would become wider with the applied pulling force. However, the temperature changing only makes the center wavelength to drift, not to spread. The neck section of FBG is etched by HF to remove the portion of cladding layer becoming to middle narrow and thick in two sides like Sandglass shape, shown in Figure 2, which has temperatureinsensitive but strain-sensitive sensor only.

When the FBG ends receiving a tensile force, the grating with whole fiber would be stretched between center point and ends, which generated a stress gradient, so that the distance of grating period would be changed along axial direction. The distance of two gratings is increased linear changing with
TABLE 1: Etching rate of HF solution with different concentration.

\begin{tabular}{lcccccc}
\hline $\mathrm{HF}: \mathrm{H}_{2} \mathrm{O}($ Molar Ratio) & $4: 1$ & $3: 1$ & $2: 1$ & $1: 1$ & $1: 2$ & $1: 3$ \\
Corrosion rate $(\mathrm{um} / \mathrm{h})$ & 4.8 & 5.75 & 10.75 & 16 & 23.2 & 49 \\
\hline
\end{tabular}

the cross-sectional area of the fiber decreased. Therefore the grating reflection bandwidth would be only broadened by applied strain and without temperature effect. So the applied strain can be obtained by measuring the output intensity see Figure 3. This method can eliminate the effect of temperature on the fiber grating, and the readout of light intensity is used to represent variation of the applied stress, which is more intuitive. The sandglass shape of FBGs is easy to preparation and measurement with photoelectric solid-state device. In the different stress and temperature of the reflected power by using the coupled mode theory calculation, when both ends of refractive index grating are constant, the reflection spectrum depends on the bandwidth of the grating length. The output intensity would change linearly with applied stress on the grating length changing but almost does not change with temperature.

In this paper, the fabrication process is given and discussed effecting with different methods. The sandglass shape of FBG is using a hydrofluoric acid solution (HF) to make isotropic etching reaction with the middle portion of optical fiber cladding material $\left(\mathrm{SiO}_{2}\right)$ :

$$
\mathrm{SiO}_{2}+6 \mathrm{HF} \longrightarrow \mathrm{H}_{2} \mathrm{SiF}_{6}+2 \mathrm{H}_{2} \mathrm{O} \text {. }
$$

The corrosion rate of fiber cladding can be controlled through adjusting the corrosion concentration, reaction time, reaction temperature, and so forth. So the sandglass shape of FBG would be easily controlled to suit for all kinds of applications. Table 1 gives the solution of variety $\mathrm{HF}$ concentrations rate. The thinner neck size of sandglass FBGs gives the better strain response, but the mechanical behavior will be declined of course. Because of that, the sandglass FBGs need each off by careful choosing the HF rate and reaction time [6].

\section{Conclusions}

The sandglass FGB sensors have a great advantage by measuring the reflected power instead of drifting center wavelength 


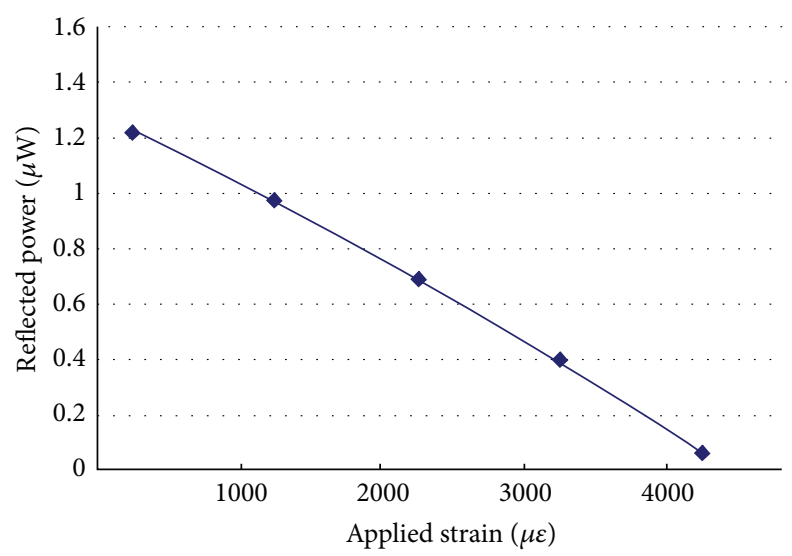

(a)

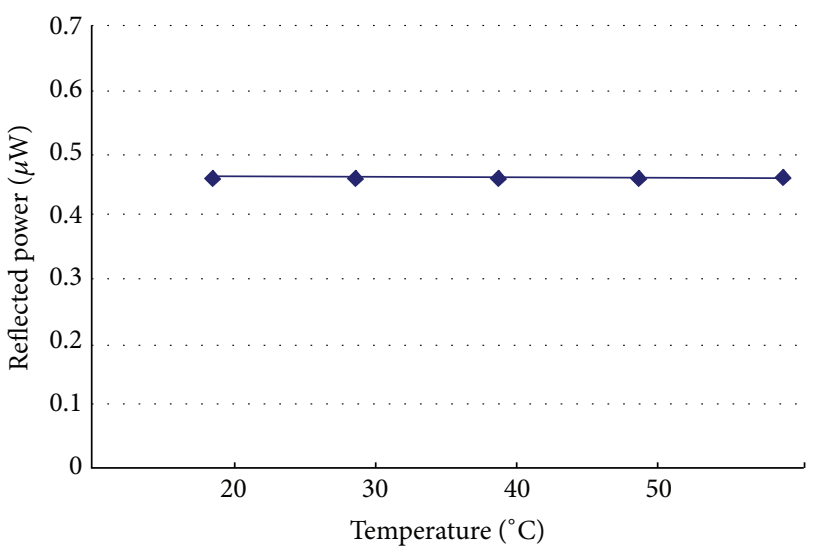

(b)

Figure 3: (a) Reflected power against applied strain; (b) reflected power against temperature.

and also solves the strain temperature in the cross-sensitivity problem at the same time without using other reference FBGs or complex demodulation system and calculating method. The sandglass shape is easy to make and control the size by corrosion concentration and etch time. But still there is mechanical strength of FBGs, which need more tests in different application and gross environment.

\section{References}

[1] M. G. Kuzyk, Polymer Fiber Optics: Materials, Physics, and Applications, CRC Press, 2006.

[2] Y. Rao, Y. Wang, and T. Zhu, Principles and Applications of Fiber Grating, Beijing Science Press, 2006.

[3] L.-B. Wan and D.-F. Wang, "Research on temperature compensation of fiber Bragg grating strain sensor based on reference grating," Journal of Optoelectronics Laser, vol. 17, no. 1, pp. 50$53,2006$.

[4] A. Othonos and K. Kalli, Fibre Bragg Gratings: Fundamentals and Applications in Telecommunications and Sensing, Artech House, Boston, Mass, USA, 1999.

[5] X. Zhang and T. Ning, "Study of cross-sensitivity in fiber grating sensors," Optical Fiber and Electric Cable, vol. 4, pp. 1-4, 2007.
[6] M. G. Xu, J.-L. Archambault, L. Reekie, and J. P. Dakin, "Discrimination between strain and temperature effects using dual-wavelength fibre grating sensors," Electronics Letters, vol. 30, no. 13, pp. 1085-1087, 1994. 

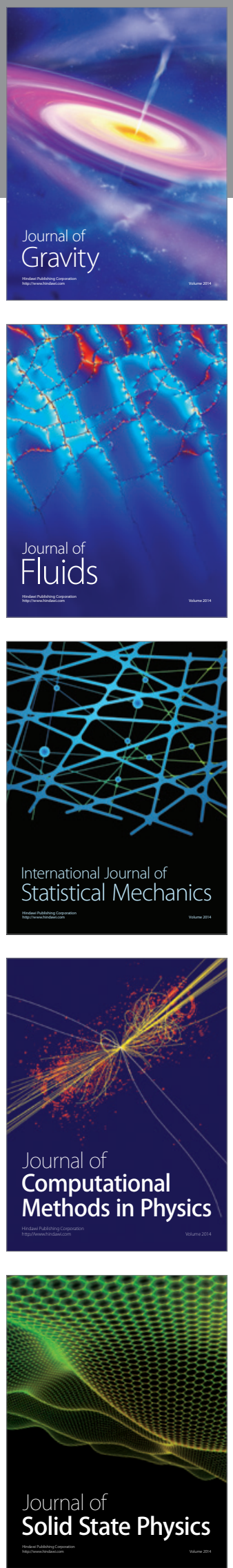

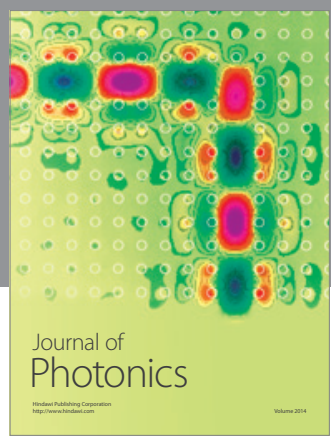

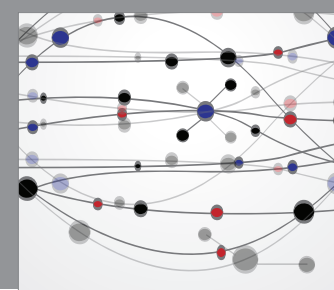

The Scientific World Journal

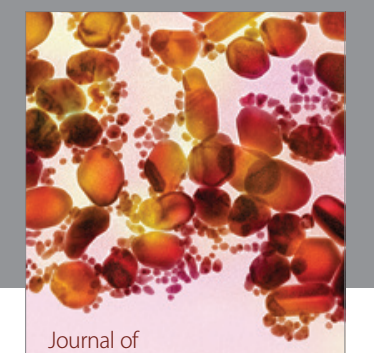

Soft Matter
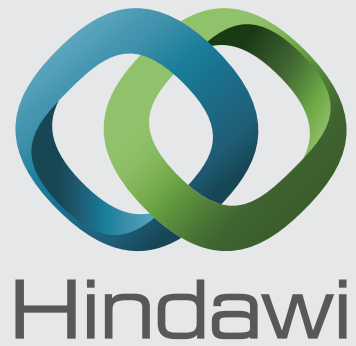

Submit your manuscripts at

http://www.hindawi.com
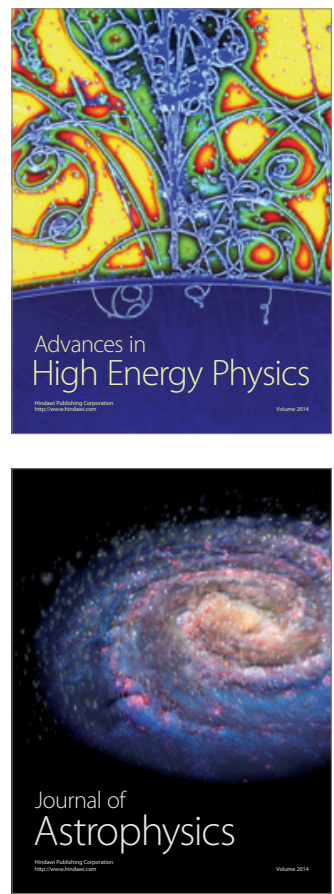
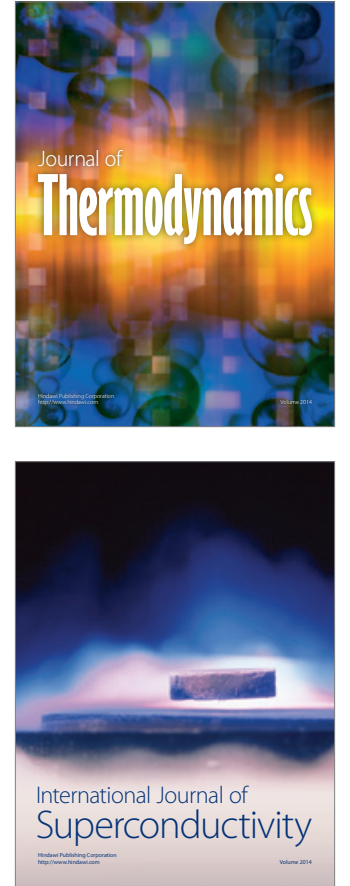
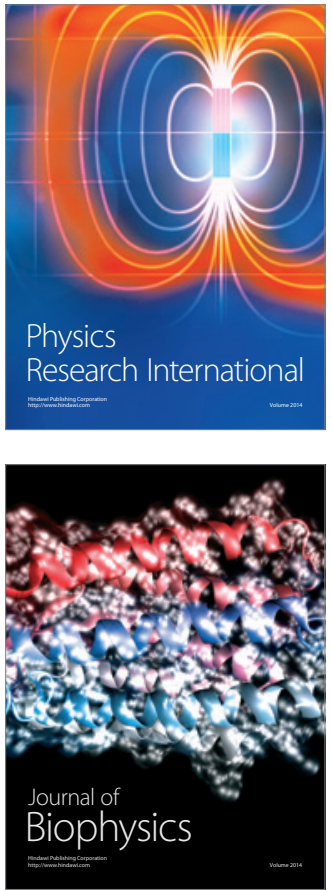
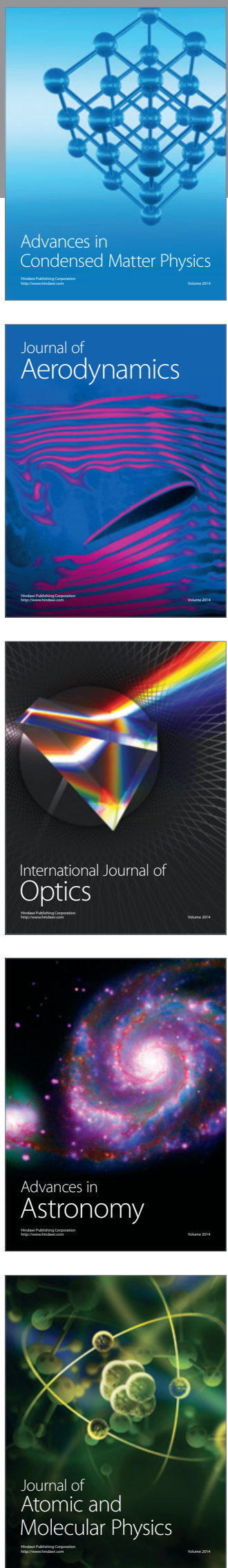